\title{
Skeletal Muscles Hypertrophy and Overweight Distinction Method in Athletes
}

\author{
Inese Pontaga \\ Department of Anatomy, Physiology, Biochemistry and Hygiene, Latvian Academy of Sports Education, LV 1006, Latvia
}

Received: January 23, 2013 / Accepted: February 05, 2013 / Published: April 25, 2013.

\begin{abstract}
The aim of the investigation is to prove that an increased body mass index (BMI) in soccer players does not always give evidence about overweight or obesity, but may appear due to skeletal muscles hypertrophy, which does not influence the players' power characteristics. Twenty nine male high qualified soccer players participated voluntary in the investigation. The anthropometrical characteristics and the body mass composition are measured by the bioelectrical impedance analysis method using the Body Composition Analyzer. Vertical jumps heights are measured on special platform. The significant correlations are determined between the BMI and the lean body mass (LM) $(r=0.36, p<0.05)$ and between the BMI and the body fat content in $\%(\mathrm{BF})(r=0.54, p<$ 0.003 ) in the soccer players. This proves that high BMI can be caused by growth of the BF and by skeletal muscles hypertrophy. The vertical jumps height is not possible to predict from the value of the BMI. Additional lean body mass due to skeletal muscles hypertrophy does not influence vertical jumps height. Relationships between the BF and jumps height are significant negative linear $(p$ $<0.013$ ). This means that increase of the BF is a reason of the decrease of vertical jump height in soccer players.
\end{abstract}

Key words: Body mass index, body bioelectrical impedance analysis method, lean body mass, fat content, soccer, vertical jump.

\section{Introduction}

Body mass index (BMI) is a useful screening tool for overweight and obesity diagnostics in children, adolescents and adults. BMI can be detected quickly and inexpensively. It is possible to calculate using the Eq. (1) [1]:

$$
\operatorname{BMI}\left(\mathrm{kg} / \mathrm{m}^{2}\right)=m / h^{2}
$$

where $m$ is a body mass in $\mathrm{kg}$; $h$ is a height of athlete in $\mathrm{m}$.

BMI correlates with adult morbidity and mortality. High BMI is associated with increased risks for hypertension, atherosclerosis, etc. [2]。 BMI gives evidence about overweight if its value in adults varies from 25 to $29.9 \mathrm{~kg} / \mathrm{m}^{2}$, but obesity can be determined if BMI is greater than $30 \mathrm{~kg} / \mathrm{m}^{2}$ [3].

Human body parts proportions and body mass composition are not taken into account if anybody

Corresponding author: Inese Pontaga, professor, M.D., Ph.D. research fields: tissue biomechanics, clinical biomechanics and sports physiology. E-mail: inese.pontaga@1spa.lv. determines BMI from tables of norms.

Human body contains different tissues, which are natural polymer materials. The main of them are muscles (35\%-50\% from the body weight), bone tissues and internal organs. These tissues together form body lean mass (LM). It provides all body movements, strength and power production. Other kind of tissues is fat, which is passive body mass. There are from $12 \%$ to $20 \%$ of fat tissue in young male's body in norm, and from $25 \%$ to $28 \%$ of fat in young female's body [1]. The body mass index depends not only on the fat content in the human body, but also on the muscles and bones mass, as well as, on the water content in the body of athletes. High value of the MBI can be estimated as overweight in athletes with great skeletal muscles mass. This means that training in many sports specializations causes increase of the body mass index [4]. High value of the BMI is observed in weight lifters, body builders, rowers, professional soccer and handball players etc.. For example, the mean body mass index in seven 
defensive linemen from a former National Football League Super Bowl team is $31.9 \mathrm{~kg} / \mathrm{m}^{2}$, but the mean BMI in all players of the team is $28.7 \mathrm{~kg} / \mathrm{m}^{2}$ [1]. However, their fat content $18 \%$ for linemen and $12.1 \%$, average for the team, misclassified them for fatness using only BMI as the overweight standard. Soccer players in the first Serbian league (elite) were taller and heavier than those in the third league (nonelite) [5]. The body mass composition in soccer players (body fat in \%, body fat mass in $\mathrm{kg}$ and fat free or lean body mass) depends on their specific role during the game: linemen, goal keepers, backs, forwards, midfielders etc. [6-9]. From the data from Wittich, et al. [10], the fat content in the body of soccer players varies from $6.1 \%$ to $19.5 \%$, and it depends on the position of the soccer player in the game: the midfielders have a significantly higher percentage of fat $(13.5 \% \pm 3.3 \%)$ than backs or forwarders $(11.0 \% \pm 2.3 \%)$. These authors determined significant positive correlation between age and fat content in the body of soccer players. Compared with age-matched general population, male adolescent soccer players (aged 12-21 year) exhibited equal body mass, higher stature, lower body mass index and lower body fat in $\%$ [11].

Body mass composition in athletes is dependent on their sport specializations. BMI in basketball players differs from its value in soccer players. For example, the mean BMI in USA National Basketball league players is in norm, only $24.5 \mathrm{~kg} / \mathrm{m}^{2}$ [1]. A large height and lean body mass due to hypertrophied skeletal muscles are beneficial for performance in adult professional team handball players. Therefore the mean height and weight increase is observed in international level handball players during the last 20-30 years [12].

The average fat content in female long distance runners' body $15.2 \%$ is significantly lower than this value in the same age sedentary females, $26 \%$ [13]. Male endurance runners have extremely low body fat content, only approximately $3 \%$. These endurance runners represent the lower end of the fat, to lean continuum for top flight athletes. It gives them some advantages in long distances running: improves heat dissipation during sport load and allows to maintain thermal balance because the isolation layer of fat tissues is thin; the greater amount of fat tissues forms passive body mass, which causes increase of energy cost to bear the additional weight.

Thickness of skinfolds in champion wrestlers is smaller than in elite wrestlers. This means that elite wrestlers have greater lean mass in the body. The mean fat content in their body is $11.0 \% \pm 4.0 \%$ (from Minnesota and Nebraska high schools) [14, 15].

Mean fat content in the body of bodybuilders is $9.3 \%$, in weight lifters, $9.1 \%$, but in the Olympic level weight lifters, $10.8 \%$ [1]. These athletes can be estimated as "overweight" using only BMI as criterion of obesity. The skeletal muscles mass in body builders is higher by $16 \mathrm{~kg}$ in comparison with the height and weight norm. The muscles mass in weight lifters is by $15 \mathrm{~kg}$ higher than in norm. All these athletes have increased body mass index due to great lean body mass.

Measurement of body mass composition is an important part of any assessment of health or fitness in different populations, as well as, in athletes. Criterion or direct methods of body composition measurement include densitometry, computed X-ray tomography (CT), magnetic resonance imaging (MRI) and dual-energy X-ray absorptiometry (DXA) [16]. These methods have high accuracy but need very expensive equipment available only in large clinics. Often checking of athletes by some of these methods (CT and DXA) are not desirable due to high doses of irradiation. All these methods are inappropriate for field and sport halls use.

Hydrostatic (underwater) weighing is generally accepted as the most reliable method for the measurement of body fat content, but is inconvenient and also not useful out of laboratory [17].

Indirect methods, including anthropometry and 
bioelectrical impedance analysis (BIA), provide estimates or indices of body composition based on results from direct or criterion methods. Indirect methods depend on biological interrelationships among direct or criterion measured body components and tissues and their distribution among normal individuals [16]. As a result, indirect methods tend to have larger predictive errors than direct methods and are affected by sample specificity and disease conditions [18].

The classic anthropometric method of body fat measurement is skinfold and circumference measures. These methods are inexpensive and non-invasive, but all measurements must be realized by qualified and experienced tester to diminish the errors of measurement. This gives possibility to estimate the fat tissue amount, but does not allow directly measurement of the skeletal muscles mass in the body. Electrical impedance analyzers have recently been proposed as an alternative to the measurement of skinfold thickness. Both these latter methods are convenient, but give values based on estimates obtained from population studies.

Body impedance analysis (BIA), a relatively simple, quick and noninvasive body composition measurement method, is reliable and easy to perform and is widely used to evaluate body composition. A small, alternating current flowing between two electrodes passes more rapidly through hydrated fat, free body tissues (lean body mass) with greater electrolyte content (lower electrical resistance) in comparison with fat tissues. As the conductance is mainly determined by the amount of water, which is only present in the fat-free mass, BIA allows calculation of the fat-free mass and $\mathrm{BF} \%$ accordingly Conference Statement, USA [19]. The analysis of body composition by bioelectrical impedance produces estimates of total body water, fat-free mass and fat mass by measuring the resistance of the body as a conductor to a very small alternating electrical current $[20,21]$. Bioelectrical impedance analyzers do not measure any biological quantity or describe any biophysical model related to obesity. Rather, the impedance index, stature (S) squared divided by resistance $(\mathrm{R}):\left(\mathrm{S}^{2} / \mathrm{R}\right)$ at a frequency, most often 50 $\mathrm{kHz}$, is proportional to the volume of total water and is an independent variable in regression equations to predict body composition [21-23]. Bioelectrical impedance analyzers use such equations to describe statistical associations based on biological relationships for a specific population, and as such the equations are useful only for subjects that closely match the reference population in body size and shape.

Maughan [17] compared values of body fat content obtained by hydrostatic weighing, skinfold thickness measurement and electrical impedance (Bodystat 500 analyser, Bodystat, Douglas, Isle of Man) on 50 healthy volunteers. Mean BF values obtained by the three methods were: hydrostatic weighing, $20.5 \% \pm$ $1.2 \%$; skinfold thickness, $21.8 \% \pm 1.0 \%$; impedance, $20.8 \pm 0.9 \%$. The results indicate that the correlation between the skinfold method and hydrostatic weighing (0.931) is somewhat higher than that between the impedance method and hydrostatic weighing (0.830). BIA equipment is developed and became more accurate in recent years. Therefore, Erceg, et al. [24] showed that mean BF percentages calculated by BIA was not significantly different from dual energy X-ray absorptiometry (DXA) and hydrostatic weighing. A good agreement was also detected between BIA and DXA by other investigators [25].

Bioelectrical impedance analysis (BIA) method was shown to be closely correlated with and as much valuable as BMI in regard to detection of obesity in healthy $21.1 \pm 1.7$ year old college students. Body fat mass and body fat percent $(\mathrm{BF} \%)$ were obtained using BIA method by hand-to-hand Omron BF-500 set, Japan. Subjects had to fast for at least $5 \mathrm{~h}$, not engage into strenuous physical activity during the previous 12 $\mathrm{h}$ and abstain from consuming caffeine beverages 
from $24 \mathrm{~h}$ before the study. The correlation between BMI values and body fat mass were 0.883 and 0.908 in males and females respectively $(p<0.001)$. Furthermore, body fat $(\mathrm{BF}) \%$ had a significant correlation with BMI in both males and females $(p<$ 0.001) [26].

There is no single universally recommended method for body composition assessment in the obese [16]. BIA has been applied to overweight or obese samples in only a few studies $[27,28]$ thus, the available BIA prediction equations are not necessarily applicable to overweight or obese children or adults. The ability of BIA to predict fatness in the obese is difficult because they have a greater proportion of body mass and body water accounted for by the trunk, the hydration of fat free body mass is lower in the obese, and the ratio of extracellular water to intracellular water is increased in the obese [27,29]. Bioelectrical impedance analysis validity and its estimates of body composition are significant issues even for normal weight individuals. BIA is useful in describing mean body composition for groups of individuals, but large errors for an individual limit its clinical application, especially among the obese. The large predictive errors inherent in BIA render it insensitive to small improvements in response to treatment [30]. Commercial bioelectrical impedance analyzers are popular and widely available to the public, but it is important to remember that these units contain all of the problems associated with this methodology. Recent BIA prediction equations have been published [31] along with body composition mean estimates for non-Hispanic whites, non-Hispanic blacks, and Mexican-American males and females from 12 to 90 years of age [32]. However, these equations are not recommended for obese individuals or groups.

The aim of the present investigation is to prove that an increased body mass index in soccer players does not always give evidence about overweight or obesity, but may appear due to skeletal muscles hypertrophy, which does not influence the players' power characteristics.

\section{Materials and Methods}

29 professional male soccer players from the team "Skonto" (Riga) participated voluntary in the investigation. Their mean age was $23.6 \pm 5.1$ years. The athletes trained in soccer from 12 to 15 years five times per week, twice per day and playing regularly in weekends. The study was performed in conformity with the standards of the ethics committee of the Latvian Council of Sciences. The measurements of football players were performed in the laboratory of the Latvian Olympic team. The standard conditions during the tests were: the air temperature in the room was $22 \pm 2{ }^{\circ} \mathrm{C}$, the measurements should be performed approximately $2 \mathrm{~h}$ after eating and within $30 \mathrm{~min}$ of voiding.

The body mass composition was measured by the bioelectrical impedance analysis method using the Body Composition Analyzer "X-Scan Plus" (Jawon Medical, Korea). Every athlete had to stay straight on the platform of the apparatus during the measurements. All athletes were weighted by the scales included in the Body Composition Analyzer. The height was measured by special ultrasound device (UHM-101) build in the Body Composition Analyzer. Body mass composition is estimated by measurement of the electrical resistance to a small, alternating current (current about $180 \mu \mathrm{A}$; multi-frequency by frequency range $1,5,50,250,550$ and $1,000 \mathrm{~Hz}$ ), flowing between four electrodes (tetra-polar method): two electrodes are positioned below the feet of every athlete and two electrodes are hold in the hands. The duration of measurement was approximately $1 \mathrm{~min}$. The obtained data were automatically processed by Dr. Lukasaki body mass composition formula [20] taking into account the height, body mass, gender, age and body tissues electrical resistance of every examined athlete. The characteristics measured and calculated by the Body Composition Analyzer are: the body mass, height, body mass index, lean body mass and fat 
content in the body in percentages. The error of measurement of the equipment was $\pm 4 \%$.

Vertical jumps heights are measured on special platform (PD. 3A, Moscow, Russia). Before the jumping test the players performed general warming up for $15 \mathrm{~min}$. Two kinds of jumps are performed: from standing position on the apparatus platform: (1) squat jump (SJ): from the standing position and before to jumping squat was performed until the knee was flexed approximately to $90^{\circ}$ and hands on hips (SJ); and (2) countermovement jump (CMJ): from the standing position and before to jumping squat was performed until the knee was flexed approximately to $90^{\circ}$ and free movements of the arms (CMJ). Every kind of jumps repeated five times, and the best results (highest SJ and CMJ) are taken into account.

The mean values and standard deviations were calculated for all determined characteristics in soccer players. The relationships between the body mass index (BMI), the body lean mass, body fat content and the height of jumps ( $\mathrm{SJ}$ and $\mathrm{CMJ}$ ) are determined using correlation and regression analysis. The relationships were considered to be statistically significant at $p<0.05$.

\section{Results}

The anthropometrical characteristics of the soccer players are: the mean height is above $180 \mathrm{~cm}(183.7 \pm$ $7.3 \mathrm{~cm})$, mean body mass is close to $80 \mathrm{~kg}(79.3 \pm 8.0$ $\mathrm{kg})$, the mean body mass index is in norm $(23.4 \pm 1.4$ $\mathrm{kg} / \mathrm{m}^{2}$ ). Only in five players the BMI exceeds 25 $\mathrm{kg} / \mathrm{m}^{2}$, which can be estimated as overweight.

Body mass composition analysis by bioelectrical impedance measurement allows us to estimate the main reasons of the BMI growth in soccer players. The body fat content in soccer players is close to the upper border of norm for males $(18.4 \pm 3.7 \%)$. The mean lean body mass is $64.6 \pm 6.1 \mathrm{~kg}$. The body fat is more than $20 \%$ from the body mass and greater in eight soccer players, but only three of them have increased BMI. Five players have normal BMI and slightly increased body fat: $21 \%-22 \%$.
The significant relationship is determined between the body mass index in the soccer players and the lean body mass (correlation coefficient $r=0.36, p<0.05$ ), Eq. (2), Fig. 1. This means that increase of the BMI in the athletes can be explained by growth of the skeletal muscles mass.

$$
\mathrm{LM}(\mathrm{kg})=26.57+1.62 \cdot \mathrm{BMI}\left(\mathrm{kg} / \mathrm{m}^{2}\right)
$$

where, $r=0.36$; standard error of the regression equation Sxy $=5.70 \mathrm{~kg} ; p<0.05$.

The similar significant relationship is detected between the body mass index and the fat content in the body in \% ( $r=0.54, p<0.003)$, Eq. (3), Fig. 2. Therefore, the increased value of BMI depends also on the greater content of fat tissue in the body.

$$
\mathrm{BF}(\%)=1.45 \cdot \mathrm{BMI}\left(\mathrm{kg} / \mathrm{m}^{2}\right)-15.55
$$

where, $r=0.54$; $\mathrm{Sxy}=3.13 \mathrm{~kg} ; p<0.003$.

The correlation between the body mass index and squat jump height is not statistically significant $(p>$ 0.05 ), as well as, the correlation between the BMI and

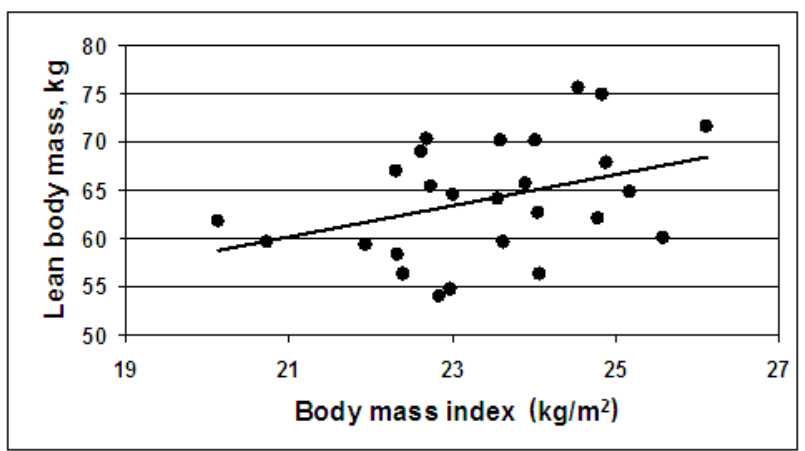

Fig. 1 Relationship between the body mass index (BMI) and lean body mass $(\mathrm{LM})$ in soccer players $(r=0.36$; $p<$ 0.05).

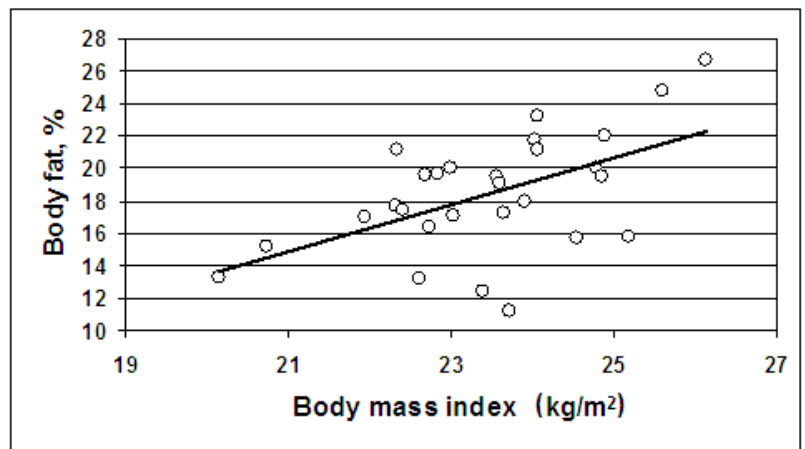

Fig. 2 Relationship between the body mass index (BMI) and body fat content (BF) in soccer players $(r=0.54 ; p<$ 0.003). 
counter - movement jump height $(p>0.05)$ in the soccer players (Fig. 3). The correlations between the lean body mass and both: squat and counter, movement jumps are not statistically significant too $(p>0.05)$, Fig. 4 This means, that the height of jumps does not depend on the skeletal muscles mass of soccer players (the main content of the lean body mass).

The relationship between the body fat (\%) and squat jump height is negative linear, Fig. 5 and the Eq. (4), as well as, the relationship between the body fat (\%) and counter-movement jump height is negative linear, Fig.5 and the Eq. (5). This means that increase of the body fat content is a reason of the decrease of vertical jump height in soccer players.

$$
\mathrm{SJ}(\mathrm{cm})=59.62-0.70 \cdot \mathrm{BF}(\%) \text {; }
$$

where, $r=-0.48$; standard error of the regression equation $\mathrm{Sxy}=4.74 \mathrm{~cm} ; p<0.013$.

$$
\mathrm{CMJ}(\mathrm{cm})=73.69-0.91 \cdot \mathrm{BF}(\%)
$$

where, $r=-0.50 ; \mathrm{Sxy}=5.70 \mathrm{~cm} ; p<0.013$.

\section{Discussion}

Our results are in agreement with the data of many other authors $[4,8,10]$ opinion concerning estimation of the body mass index value with caution in athletes. The high value of the BMI can be estimated as overweight in athletes with skeletal muscles hypertrophy because the body mass index depends not only on the body fat content, but also on skeletal muscles mass. From our data: the significant correlation is determined between the body mass index and the lean body mass $(r=0.36, p<0.05)$, as well as, between the BMI and the body fat content in $\%(r=0.54, p<0.003)$ in the soccer players. This means that the muscles mass and the body fat content both are important in BMI determination in soccer players.

The body fat content depends on inheritance: the non-Caucasian players demonstrated significantly lower percent body fat $(9.2 \pm 2.0 \%)$ than the Caucasian players $(10.7 \% \pm 1.8 \%)$ [9]. The body mass composition in soccer players depends on their specific role during the game (linemen, goal keepers, backs, forwards and midfielders, etc.) [6-9]. The midfielders have a significantly higher percentage of fat than backs or forwarders [10]. The body mass index

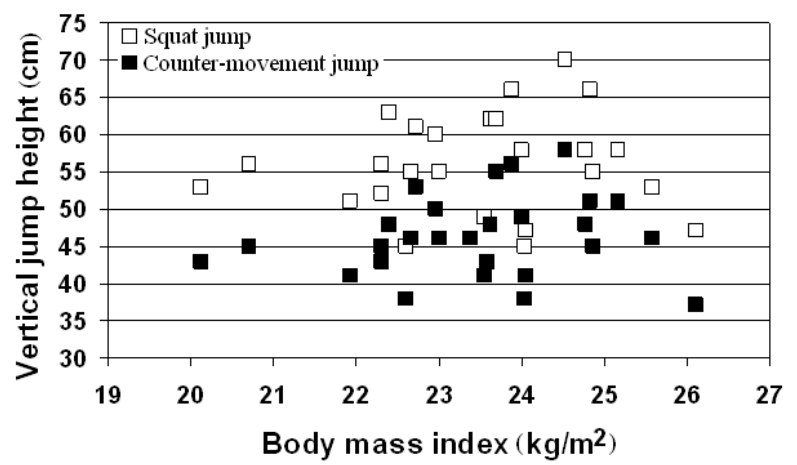

Fig. 3 Relationship between the body mass index in the soccer players and the vertical jump height: squat jump, coefficient of correlation $r=0.14, p>0.05$; counter-movement jump, $r=\mathbf{0 . 0 8}, p>0.05$.

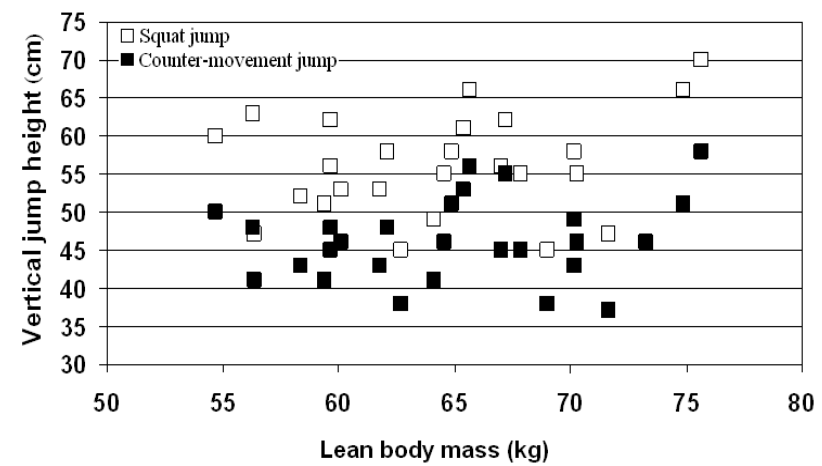

Fig. 4 Relationship between the lean body mass in the soccer players and the vertical jump height: squat jump, coefficient of correlation $r=0.20, p>0.05$; counter-movement jump, $r=0.24, p>0.05$.

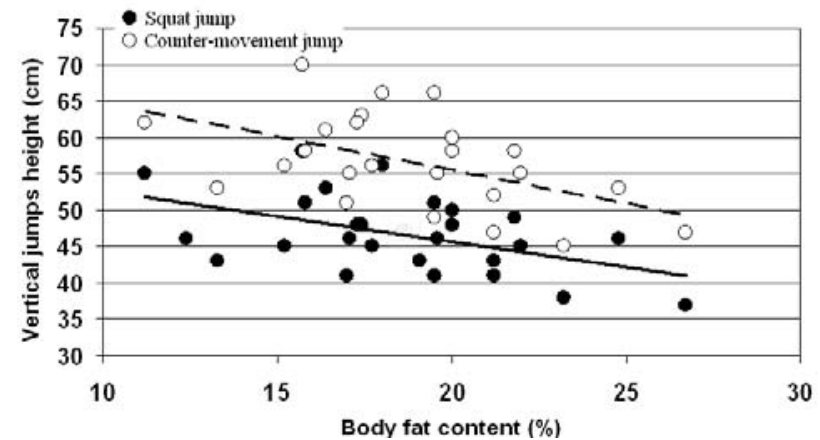

Fig. 5 Relationships between the body fat content and

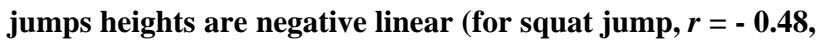
$p<0.013$ and for counter-movement jump, $r=-0.50, p<$ 0.013). 
$\left(23.4 \pm 1.4 \mathrm{~kg} / \mathrm{m}^{2}\right)$ is in norm and body fat content $(18.4 \% \pm 3.7 \%)$ is close to the upper border of norm in Latvian highly qualified soccer players. This can be explained by the young age of our players $(23.6 \pm 5.1$ years) in comparison with elite international level soccer players, because the significant positive correlation between age and fat content in the body of soccer players is determined [10].

The significant correlation between the BMI and the height of vertical jumps (squat jump and counter, movement jump) is not determined in high qualification soccer players $(p>0.05)$. This means that the vertical jumps height is not possible to predict from the value of the BMI. The main reason of the BMI increase is skeletal muscles mass growth or hypertrophy due to long term regularly training in soccer. This allows maintain the same vertical jump height in soccer players with smaller or larger body mass. Relationships between the body fat content and jumps heights are significant negative linear. This means that overweight of soccer players due to growth of fat content causes decrease of their jumping performance and players must avoid it.

Although there is some skepticism about using of bioelectrical impedance systems in athletes body mass composition measurement, this method is enough accurate for normal subjects, especially in the field of sports medicine, in addition to the reliable conventional anthropometric methods for evaluation of physical fitness. The errors of measurement are small and systemic. For example, Kim et al. [33] compared the body fat \% measurements by direct dual-energy X-ray absorptiometry (DEXA) method and the eight electrode bioelectrical impedance (BIA) method in 174 healthy Korean adults. The mean difference between BIA \% fat and DEXA \% fat was small but significant $(p<0.05)$, which resulted in an overestimation of $1.2 \% \pm 2.2 \%$ fat in men and an underestimation of $2.0 \% \pm 2.4 \%$ fat in women. However, the eight-electrode BIA had small, but systemic, errors of $\%$ fat in the predictive accuracy for individual estimation. The total errors led to an overestimation of fat percentages in lean men and an underestimation in obese women. Therefore, body composition results assessed by BIA in elite athletes should be interpreted with caution, especially in individual subjects with expressed skeletal muscles hypertrophy or high body fat content. Nevertheless, all above mentioned BIA allows distinguish muscles hypertrophy from fatness in high qualified soccer players. From our data: BMI exceeds $25 \mathrm{~kg} / \mathrm{m}^{2}$, which can be estimated as overweight in five Latvian soccer players, but only three of them have body fat content above the norm (20\%). Therefore, body mass composition analysis by bioelectrical impedance measurement allows us to estimate the main reasons of the BMI growth in soccer players.

The body composition and its variation in seasons can affect the performance of soccer players. Bioelectrical impedance vector analysis allows estimate seasonal variations in body composition in elite soccer players at least in terms of variation of soft tissues (mass and hydration) [34]. Therefore, BIA method is useful as a screening tool for body mass composition changes control in soccer players during long time period. If body fat content increased, it is possible to reduce the fat by exercises and special diets.

\section{Conclusions}

The significant correlation is determined between the body mass index and the lean body mass $(r=0.36$, $p<0.05$ ), as well as, between the BMI and the body fat content in percentages $(r=0.54, p<0.003)$ in the soccer players. This means that high body mass index can be caused by growth of the body fat content and by increased skeletal muscles mass (muscles hypertrophy).

The body mass index mean value $\left(23.4 \pm 1.4 \mathrm{~kg} / \mathrm{m}^{2}\right)$ is in norm and body fat content $(18.4 \% \pm 3.7 \%)$ is close to the upper border of norm in highly qualified soccer players. BMI exceeds $25 \mathrm{~kg} / \mathrm{m}^{2}$ in five soccer 
players, but only three of them have body fat content above the norm (20\%). Therefore body mass composition analysis by bioelectrical impedance measurement allows us to distinguish muscles mass growth from fatness in soccer players.

The correlations between the body mass index or the lean body mass and the height of vertical jumps (squat jump and counter, movement jump) are none significant in soccer players $(p>0.05)$. This proves that the vertical jumps height is not possible to predict from the value of the body mass index. Additional lean body mass due to skeletal muscles hypertrophy does not influence vertical jumps height.

Relationships between the body fat $\%$ and jumps heights are significant negative linear (for squat jump: $r=-0.48, p<0.013$, and for counter, movement jump: $r=-0.50, p<0.013)$. This means that increase of the body fat content is a reason of the decrease of vertical jump height in soccer players.

\section{References}

[1] W.D. McArdle, F.I. Katch, V.L. Katch, Body Composition: Components, Assessment, and Human Variability, Essentials of Exercise Physiology, Lippincott Williams and Wilkins, USA, 2000, pp. 500-527.

[2] K.A. Witt, E.A. Bush, College athletes with an elevated body mass index often have a high upper arm muscle area, but not elevated triceps and subscapular skinfolds, Journal of American Diet Association 105 (2005) 599-602.

[3] E.M. Mathews, D.R. Wagner, Prevalence of overweight and obesity in collegiate American football players by position, Journal of American College of Health 57 (1) (2008) 33-38.

[4] J.J. Ode, J.M. Pivarnik, M.J. Reeves, J.L. Knous, Body mass index as a predictor of percent fat in college athletes and nonathletes, Medicine and Science in Sports and Exercise 39 (3) (2007) 403-409.

[5] S.M. Ostojic, Elite and nonelite soccer players: Preseasonal physical and physiological characteristics, Research in Sports Medicine 12 (2004) 143-150.

[6] C. Carling, E. Orhant, Variation in body composition in professional soccer players: Interseasonal and intraseasonal changes and the effects of exposure time and player position, Journal of Strength and Conditioning Research 24 (2010) 1332-1339.

[7] T. Hazir, Physical characteristics and somatotype of soccer players according to playing level and position, Journal of Human Kinetics 26 (2010) 83-95.

[8] G. Melchiorri, G. Monteleone, A. Andreoli, C. Calla, M. Sgroi, A.D. Lorenzo, Body cell mass measured by bioelectrical impedance spectroscopy in professional football (soccer) players, Journal of Sports Medicine and Physical Fitness 47 (2007) 408-412.

[9] L. Sutton, M. Scott, J. Wallace, T. Reilly, Body composition of English Premier League soccer players: Influence of playing position, international status, and ethnicity, Journal of Sports Sciences 27 (10) (2009) 1019-1026.

[10] A. Wittich, B. Oliveri, E. Rotemberg, C. Mautalen, Body composition of professional football (soccer) players determined by dual X-ray absorptiometry, Journal of Clinical Densitometry 4 (2001) 51-55.

[11] P.T. Nikolaidis, N.V. Karydis, Physique and body composition in soccer players across adolescence, Asian Journal of Sports Medicine 2 (2) (2011) 75-82.

[12] E.M. Gorostiaga, C. Granados, J. Ibanez, M. Izquerdo, Differences in physical fitness and throwing velocity among elite and amateur male handball players, International Journal of Sports Medicine 26 (2005) 225-232.

[13] M.L. Pollock, L.R. Guttman, A. Jackson, J. Ayres, A. Ward, A.C. Linnerud, Body composition of elite class distance runners, Annals of New York Academy of Sciences 301 (1977) 361-370.

[14] K.S. Clarke, Predicting certified weight of young wrestlers, a field study of the Tcheng-Tipton methods, Medicine and Science in Sports 6 (1974) 52-57.

[15] T.J. Housh, G.0. Johnson, K.B. Kenney, S.L. McDowell, R.A. Hughes, C.J. Cisar, et al., Validity of anthropometric estimations of body composition in high school wrestlers, Research Quarterly in Exercise and Sport 60 (1989) 239-245.

[16] D.L. Duren, R.J. Sherwood, S.A. Czerwinski, M. Lee, A.C. Choh, R.M. Siervogel, et al., Body composition methods: Comparisons and interpretation, Journal of Diabetes Science and Technology 2 (6) (2008) 1139-1148

[17] R.J. Maughan, An evaluation of a bioelectrical impedance analyzer for the estimation of body fat content, British Journal of Sports Medicine 27 (1) (1993) 63-66.

[18] W.M. Chumlea, S.S. Guo, Assessment and prevalence of obesity: Application of new methods to a major problem, Endocrinology 13 (2) (2000) 135-142.

[19] Bioelectrical impedance analysis in body composition measurement, in: National Institutes of Health Technology Assessment Conference Statement, American Journal of Clinical Nutrition 64 (1996) 524S-532S. 
[20] H.C. Lukaski, P.E. Johnson, W.W. Bolonchuk, G.I. Lykken, Assessment of fat-free mass using bioelectrical impedance measurements of the human body, American Journal of Clinical Nutrition 41 (4) (1985) 810-817.

[21] R.N. Baumgartner, W.C. Chumlea, A.F. Roche, Bioelectric impedance for body composition, Exercise and Sports Sciences Reviews, MacMillan, New York, 1990, pp. 193-224.

[22] W.C. Chumlea, S.S. Sun, Bioelectrical Impedance Analysis, Human Body Composition, Champaign, IL: Human Kinetics Books, 2005.

[23] S.S. Sun, W.C. Chumlea, Statistical methods for the development and testing of body composition prediction equations, Human Body Composition, Champaign, IL: Human Kinetics Books, 2005.

[24] D.N. Erceg, C.M.D. Conwright, A.E. Rossuello, N.E. Jensky, S. Sun, E.T. Schroeder, The stayhealthy bioelectrical impedance analyzer predicts body fat in children and adults, Nutrition Research 30 (2010) 297-304.

[25] N. Gupta, G. Balasekaran, V. Govindaswamy, C.Y. Hwa, L.M. Shun, Comparison of body composition with bioelectric impedance (BIA) and dual energy X-ray absorptiometry (DEXA) among Singapore Chinese, Journal of Science and Medicine in Sport 14 (1) (2011) 33-35.

[26] S.T. Heydari, S.M.T. Ayatollahi, N. Zare, Diagnostic value of bioelectrical impedance analysis versus body mass index for detection of obesity among students, Asian Journal of Sports Medicine 2 (2) (2011) 68-74.

[27] D.S. Gray, G.A. Bray, N. Gemayel, K. Kaplan, Effect of obesity on bioelectrical impedance, American Journal of Clinical Nutrition 50 (2) (1989) 255-260.
[28] R.F. Kushner, A. Kunigk, M. Alspaugh, P.T. Andronis, C.A. Leitch, D.A. Schoeller, Validation of bioelectrical impedance analysis as a measurement of change in body composition in obesity, American Journal of Clinical Nutrition 52 (2) (1990) 219-223.

[29] W.C. Chumlea, Body Composition Assessment of Obesity, Overweight and the Metabolic Syndrome: From Bench to Bedside, Springer, New York, 2006, pp. 23-35.

[30] G. Forbes, Human Body Composition, Growth, Aging, Nutrition, and Activity, Springer-Verlag, New York, 1987.

[31] S.S. Sun, W.C. Chumlea, S.B. Heymsfield, H.C. Lukaski, D. Schoeller, K. Friedl, et al., Development of bioelectrical impedance analysis prediction equations for body composition with the use of a multicomponent model for use in epidemiological surveys, American Journal of Clinical Nutrition 77 (2) (2003) 331-340.

[32] W.C. Chumlea, S.S. Guo, R.J. Kuczmarski, K.M. Flegal, C.L. Johnson, S.B. Heymsfield, et al., Body composition estimates from NHANES III bioelectrical impedance data, International Journal of Obesity and Relative Metabolic Disorders 26 (12) (2002) 1596-1609.

[33] H. Kim, C.H. Kim, D.W. Kim, M. Park, H.S. Park, S.S. Min, et al., External cross-validation of bioelectrical impedance analysis for the assessment of body composition in Korean adults, Nutrition Research and Practice 5 (3) (2011) 246-252.

[34] A. Bonuccelli, F. Marzatico, G. Stesina, L. Stefanini, D. Buonocore, S. Rucci, et al., Bioelectrical impedance vector analysis (BIVA) to evaluate seasonal variations in body composition of elite soccer players, Journal of the International Society of Sports Nutrition 8 (Suppl. 1) (2011) 37 . 